\title{
Consecuencias de la resección de la cabeza radial sobre la articulación radiocubital distal ${ }^{*}$
}

\author{
H. Domínguez Martínez ${ }^{(1)}$, J. Goyeneche Paternain ${ }^{(2)}$, I. Torre Hernández ${ }^{(2)}$ \\ Hospital Donostia. San Sebastián \\ (1) Médico Adjunto De Cirugía Plástica \\ (2) Médico adjunto De Traumatología
}

Correspondencia:

Dra. Haroa Domínguez Martínez

Hospital Donostia

Paseo Dr. Beguiristain SN

San Sebastián (Guipúzcoa)

Email: haroadom@yahoo.es

Teléfono: 943007000

\begin{abstract}
Nuestro objetivo ha sido la realización de un estudio clínico y radiológico de las secuelas en la articulación radiocubital distal en pacientes intervenidos de una extirpación aislada, total e inmediata, de cabeza radial, tras haber sufrido una fractura de tipo III y IV de Mason. Para ello hemos estudiado a 45 pacientes intervenidos en el Hospital Donostia entre los años 1988 y 2004, a los que se les ha realizado una entrevista clínica, una exploración física y radiológica del brazo intervenido, así como del contralateral para realizar el estudio comparativo. Hemos encontrado diferencias estadísticamente significativas en la medición de fuerza prensil, laterodigital y pulpar, y en la existencia de cambios degenerativos radiográficos. Observamos también disminución en los arcos de movilidad de la muñeca siendo los movimientos de supinación y extensión los más afectados $\left(1,9^{\circ}\right.$ y $2,55^{\circ}$ respectivamente de media respecto al contralateral). El valor medio de dolor en la muñeca intervenida en una escala analógica visual fue de 0,13 en reposo y 0,65 en actividad. No encontramos trastornos neurológicos, y los pacientes expresaron un alto grado de satisfacción con el resultado. Por to-
\end{abstract}

The purpose of this study was to evaluate the clinical and radiological side-effects of iolated, total and immediate radial head resection on distal radio-ulnar joint (DRUJ) after Mason's type III and IV fracture. We studied 45 patients that were operated at Donostia Hospital between 1988 and 2004 and we interviewed them personally, explored them physically and X-rayed both of their operated and non-operated elbows and wrists to cnpare them. We found statistically significant differences between the operated and the non-operated extremities in terms of grip strength, side to side pinch strength and fine pinch strength and in radiological degenerative changes. We also noticed that there was a decrease in wrist's movements and that supination and extension were the most affected ones $\left(1,9^{\circ}\right.$ and $2,55^{\circ}$ respectively of mean difference with the healthy side)The mean value of pain in wrist on an Analogic Visual Scale was 0,13 at rest and 0,65 when moving. We didn't find any neurologic changes and the patients were highly satisfied with their present condition. Therefore, we concluded that even there is statistical diference, this doesn't interfere with

\footnotetext{
* El presente estudio recibió el premio a la mejor comunicación del VII encuentro Iberolatinoamericano de cirugía de la mano. XVIII Congreso Nacional de la SECMA celebrado en Zaragoza en el 2007.
} 
do ello podemos concluir que aunque hay diferencia estadística, ésta no parece interferir en la realización de actividades de la vida diaria de nuestros pacientes ni por disminución de fuerza o movilidad ni por presencia de dolor.

Palabras clave: cabeza radial, excisión, articulacion radiocubital distal. the daily living activities of our patients because of decrease of strength or for

Key words: radius head, excision, distal radioulnar joint.

\section{INTRODUCCIÓN}

厂 a extirpación de la cabeza radial es un procedimiento ampliamente utilizado como tratamiento de determinadas fracturas de cabeza radial, pero que no es aceptado por todos los especialistas debido a las secuelas que pueden aparecer en la articulación del codo y de la muñeca. Según la clasificación de Mason las fracturas de cabeza radial se clasifican en 4 tipos de menor a mayor gravedad siendo las de tipo III y IV las que presentan mayor controversia en cuanto al tratamiento óptimo, debido a que habitualmente son fracturas complejas asociadas a lesiones ligamentosas ${ }^{1}$. Las posibles opciones terapéuticas consisten en la fijación interna ${ }^{2-7}$, la extirpación de la cabeza radial $^{8-10}$, o la artroplastia de sustitución $^{11-13}$. En la mayoría de los casos la fijación interna se convierte en una ardua tarea por la gran conminución de los fragmentos y la posterior necrosis de los mismos por devascularización. En cuanto a la resección de la cabeza radial, existen algunos estudios en cadáver, que indican el importante papel de la misma en la estabilidad del codo sobretodo en el varo y la rotación externa forzada $^{14}$, más aún si existe lesión de otros elementos estabilizadores de esta articulación como el ligamento colateral medial ${ }^{15-18}$. Por otro lado las prótesis de sustitución, bien de silicona o metálicas, tampoco son comúnmente aceptadas ${ }^{19}$. No existen estudios con un número importante de pacientes, ni con resultados estadísticamente significativos, que demuestren sus ventajas respecto a la escisión de la cabeza radial.

Si nos centramos en la articulación radiocubital distal, la mayoría de las secuelas que se recogen en la literatura tras la realización de la resección de la cabeza radial son debidas a la migración proximal que puede sufrir el hueso tras dicha intervención ${ }^{20}$. Esta migración -en la que tiene un importante papel la membrana interósea $^{21}$ - se manifiesta en la mayoría de los casos como dolor crónico de muñeca, que afecta sobretodo a la región cubital por impactación del extremo distal del cúbito sobre el carpo, lesión por otra parte de difícil solución ${ }^{22}$. También se han descrito compresiones del nervio interóseo anterior, que se manifiesta por dolor y parálisis o paresia del flexor digitorum profundus de $2^{\circ} \mathrm{y}$ $3^{\circ}$ dedo, del flexor pollicis longus y del pronator teres. Incluso Crawford et al. ${ }^{23}$ reportaron dos casos de síndrome del túnel radial.

Para evaluar nuestros resultados con esta técnica, ante la escasez de opciones terapéuticas, decidimos realizar un estudio cuyo objetivo ha sido evaluar clínica y radiológicamente la articulación radio-cubital distal (RCD) de pacientes intervenidos de este tipo de fracturas.

\section{MATERIAL Y MÉTODO}

Hemos realizado un estudio de cohortes retrospectivo donde el factor de exposición es haber sido intervenido de una extirpación de cabeza radial tras una fractura aislada de cabeza radial, y donde tomaremos como no expuestos el brazo contralateral que se supone sano.

Entre 1988 y 2004 fueron intervenidos de una extirpación de cabeza radial 72 pacientes en el Hospital Donostia de San Sebastián, sin embargo no todos pudieron ser localizados y tres de ellos habían fallecido. Tras el cumplimiento de los criterios de selección que habíamos fijado, nuestra muestra de pacientes se redujo finalmente a 45 pacientes. Los criterios de selección consistían en 
la presentación de una fractura aislada de cabeza radial, es decir, no presentar fracturas ni luxaciones concomitantes en ese brazo, ni otro tipo de fracturas o luxaciones en el brazo contralateral que nos impidieran una correcta valoración de los parámetros clínicos o radiológicos. También excluimos pacientes que habían presentado una extirpación de cabeza radial diferida o parcial, y aquellos a los que se les había practicado la intervención en un periodo de tiempo inferior a 18 meses para que pudiéramos valorar secuelas a medio-largo plazo. La muestra final de 45 pacientes estaba constituida por el $62,2 \%$ mujeres (28 casos) y con una media de edad de 54,51 años $(r=22-85)$. Prácticamente la serie de pacientes con fractura del tipo III $(51,1 \%)$ y del tipo IV (49,9\%) eran iguales. El 75,6\% había sufrido la fractura mediante un traumatismo de baja energía por caída con codo en extensión. El lado derecho fue el afectado en el 75,6\% de los pacientes, y resultó ser el dominante en el 91,1\%. El tiempo medio de seguimiento fue de 109,47 meses (DE 53,41) $(r=21-212)$.

Los pacientes fueron revisados en consulta donde se les realizó una entrevista, una exploración física y una serie de radiografías del codo y muñeca del lado sano y del intervenido. Clínicamente medimos una serie de variables tales como la presencia de dolor mediante una escala analógica visual del 0 al 10, tanto en reposo como en actividad, la fuerza prensil, laterodigital y pulpar mediante dinamómetro Jamar y el arco de movimientos de la muñeca comparándolo con el contralateral mediante un goniómetro. También valoramos variables que nos indicaran una posible lesión neurológica como la presencia de signo de Tinel en el canal epitroclear, y la sensibilidad de los dedos mediante el Test de discriminación de puntos de Weber.
Radiológicamente medimos la varianza cubital valorando la diferencia respecto al lado sano, la presencia de cambios degenerativos en el codo en 3 grados de gravedad según la clasificación de Broberg y Morrey ${ }^{21}$, y la cantidad de hueso radial extirpado tomando como punto de referencia el inicio de la tuberosidad radial hasta la epífisis proximal del radio en el lado sano y comparándolo con el intervenido.

Finalmente también les interrogamos sobre su grado de satisfacción con la intervención y su estado actual. Los datos obtenidos fueron analizados para la búsqueda de resultados estadísticamente significativos.

\section{RESULTADOS}

Los pacientes no referían en general dolor en la muñeca ni en el codo sano, frente a una media de intensidad de dolor en el codo intervenido en reposo de 0,84 (DE 1,3) y en actividad de 1,44 (DE 1,8$)$, y en muñeca de 0,13 (DE 0,4 ) y 0,65 $(\mathrm{DE} 0,9)$ respectivamente (medido en una escala del 0 al 10).

En cuanto a la amplitud de movimientos en la articulación radiocubital distal comparándola con el lado sano, no intervenido, no se detectaron grandes diferencias. La media de grados de déficit de pronación y supinación comparados con el brazo sano fueron de 0,89 (DE 2,9) y 1,89 (DE 5,3) respectivamente mientras que de flexoextensión fueron 0,33 (DE 1,7) y 2,55 (DE 4,8).

El $24,4 \%$ de los pacientes (11 casos) presentaron Tinel en el canal epitroclear, pero ningún paciente refirió hipoestesias o parestesias en los territorios inervados por el nervio mediano o cubital, y los test de medición de sensibilidad epicrítica aplicados no detectaron anomalías.

TABLA I - COMPARACIÓN DE FUERZA LADO SANO/INTERVENIDO

\begin{tabular}{|l|c|c|c|}
\hline & MEDIA & IC (95\%) INF & IC (95\%) SUP \\
\hline Fuerza prensil S/E & 2'8 & 1'07 & 4'49 \\
\hline Fuerza pulpar S/E & 0'4 & 0'11 & 0'56 \\
\hline Fuerza laterodigital S/E & 0'6 & 0'27 & 0'93 \\
\hline
\end{tabular}




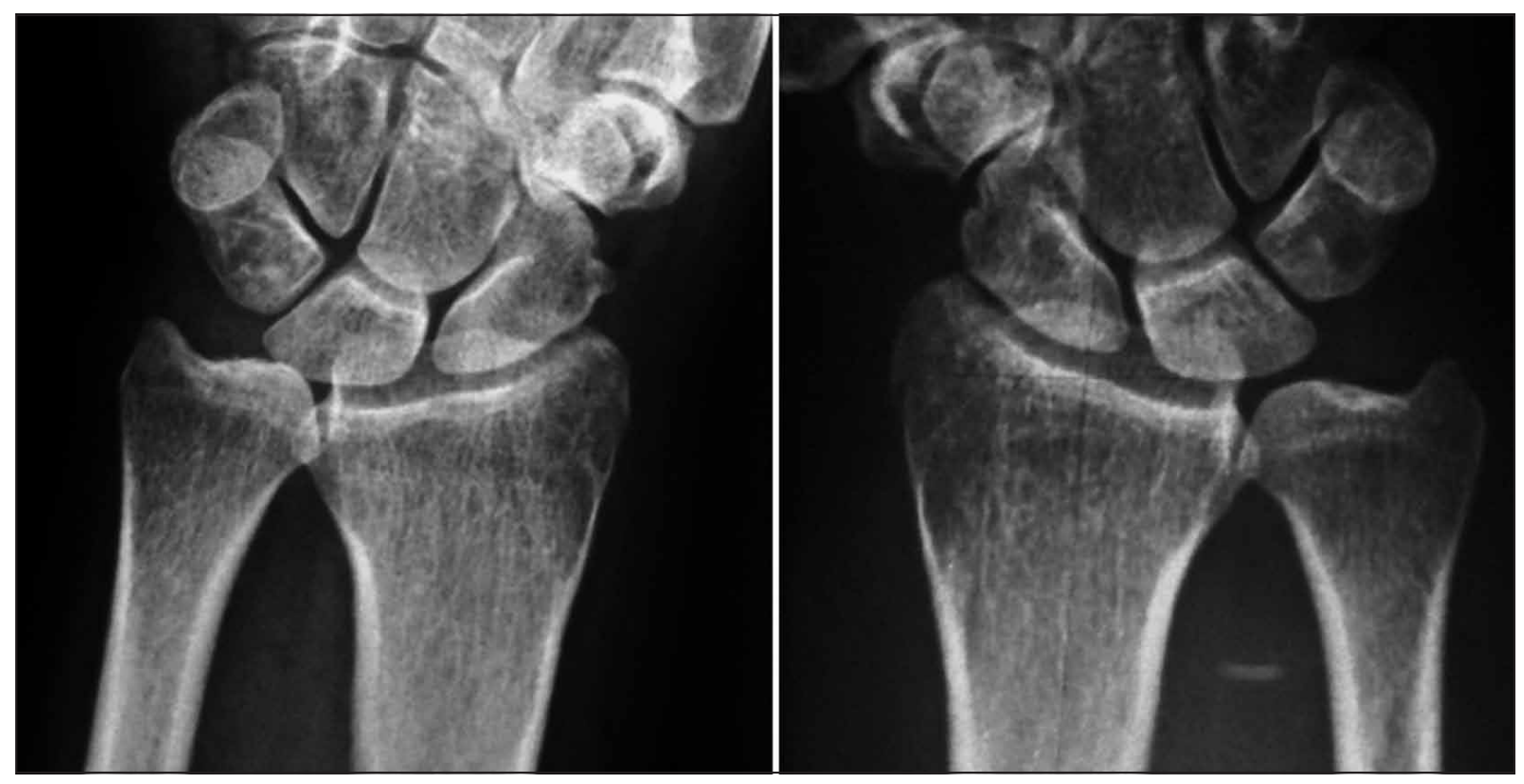

Figura 1. Cúbito plus.

Las diferencias más importantes las hayamos en cuanto a la medición de fuerza en el brazo intervenido. La media de fuerza prensil fue de $31,80 \mathrm{~kg}(\mathrm{DE} \mathrm{10,47)}$ en el lado sano frente a $29,02 \mathrm{~kg}$ (DE 11,85) en el brazo intervenido; la fuerza pulpar de $4,4 \mathrm{~kg}$ (DE 1,88) frente a $4 \mathrm{~kg}$ (DE 1,56) y la laterodigital de 7,2 $\mathrm{kg}$ (DE 2,56) frente a $6,6 \mathrm{~kg}$ (DE 2,6), siendo todas estas diferencias estadísticamente significativas con una $\mathrm{p}<0,05$ (Tabla I).

En cuanto a las mediciones radiológicas la media de resección radial fue de 10,78 mm (DE 2,6) observándose una media de varianza cubital 1,18 mm (DE 0,912) (Figura 1).

Se observaron cambios degenerativos importantes (grados 2 y 3 ) en el $40 \%$ de los codos in- tervenidos frente al $6,7 \%$ de los codos sanos, así como en $15,5 \%$ de las muñecas del brazo afecto mientras que no vimos ningún caso de artrosis grave en ninguna muñeca sana. Estas diferencias fueron estadísticamente significativas con una $\mathrm{p}<0,05$ (Tabla II).

Aunque no era el objetivo de nuestro estudio observamos que gran parte de los pacientes presentaban un valgo del codo intervenido que varió de $0^{\circ}$ a $35^{\circ}$ con una media de $12,76^{\circ}$ con una DE de 7,44 y un déficit de extensión del mismo de $12,22^{\circ}$ con una DE de 7,27 en un rango de $0^{\circ}$ a $30^{\circ}$.

Finalmente los pacientes fueron encuestados en cuanto a su grado de satisfacción (del 0 al 10) con los resultados de la intervención a la

\section{Tabla II - Cambios degenerativos Radiológicos}

\begin{tabular}{|l|c|c|c|c|}
\hline & $\begin{array}{c}\text { GRADO } \\
\mathbf{0}(\boldsymbol{\%})\end{array}$ & $\begin{array}{c}\text { GRADO } \\
\mathbf{1}(\boldsymbol{\%})\end{array}$ & $\begin{array}{c}\text { GRADO } \\
\mathbf{2}(\mathbf{\%})\end{array}$ & $\begin{array}{c}\text { GRADO } \\
\mathbf{3}(\boldsymbol{\%})\end{array}$ \\
\hline CODO LADO SANO & 80 & $13^{\prime} 3$ & $6^{\prime} 7$ & 0 \\
\hline CODO INTERVENIDO & $28^{\prime} 9$ & $31^{\prime} 1$ & $24^{\prime} 4$ & $15^{\prime} 6$ \\
\hline MUÑECA LADO SANO & $91^{\prime} 1$ & $8^{\prime} 9$ & 0 & 0 \\
\hline MUÑECA INTERVENIDA & $77^{\prime} 8$ & $6^{\prime} 7$ & $13^{\prime} 3$ & 2 '2 \\
\hline
\end{tabular}




\section{Tabla III - Grado de SAtisfacción}

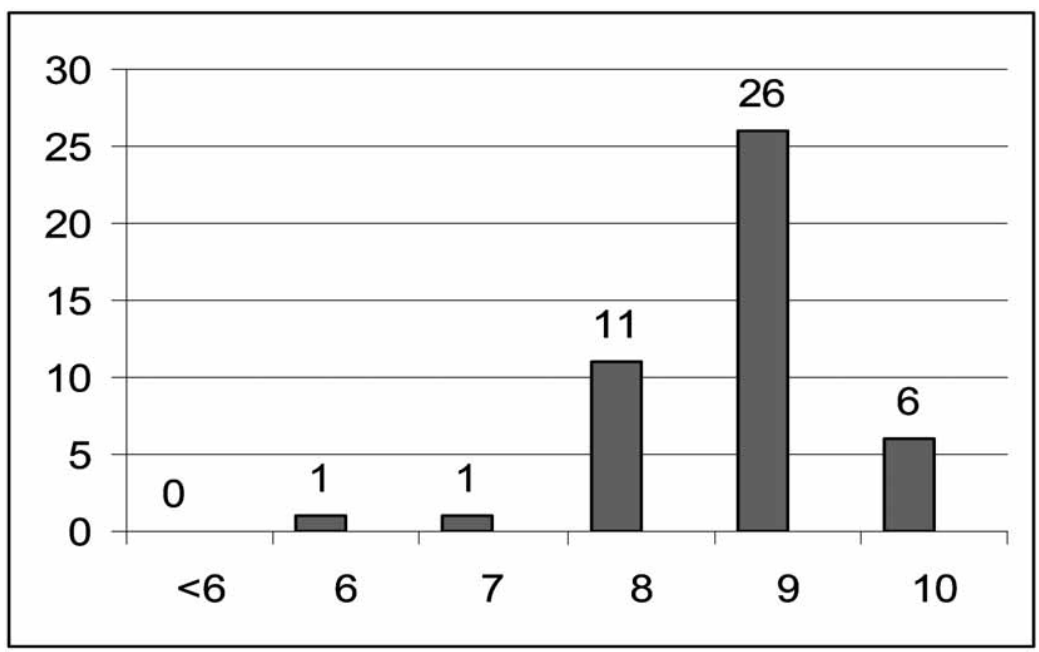

que fueron sometidos y los resultados fueron los expuestos en la Tabla III pudiendo afirmar que el 97,8\% darían una calificación de más de 7 .

\section{DISCUSIÓN}

A pesar de las de complicaciones que se recogen en la literatura tras una extirpación de cabeza radial lo cierto es que a la luz de los resultados de nuestro estudio no podemos concluir que dicha intervención deje importantes secuelas en la extremidad en general y menos aún en la articulación radiocubital distal.

Sí que es cierto que se observan diferencias estadísticamente significativas en cuanto a la pérdida de fuerza de la mano, pero si nos fijamos en los valores numéricos se aprecia que la magnitud de la diferencia es pequeña. Es destacable que en el 91,1\% de los casos el brazo afecto era el dominante y que no hemos efectuado ningún tipo de corrección pero, aún suponiendo que los valores de las diferencias sean mayores, es difícil que una pérdida de media de 1 a $4,5 \mathrm{~kg}$ de fuerza prensil y menos aún de 0,14 a $0,5 \mathrm{~kg}$ de fuerza pulpar afecte a las actividades de vida diaria de nuestros pacientes tales como abrir un bote o utilizar un destornillador.

También observamos diferencias estadísticamente significativas en cuanto a cambios de- generativos en la radiografía, sobretodo en la articulación radiohumeral como es lógico, pero clínicamente estos no se traducen en dolores importantes ya que la media de dolor que refirieron los pacientes en las muñecas intervenidas fue de 0,65 en actividad, siendo aún menor en reposo con una media de 0,13 . Estas alteraciones radiológicas tampoco se manifiestan en una disminución considerable de la movilidad de la articulación radiocubital distal pues los valores medios de limitación de los movimientos de supinación y extensión fue de unos $2^{\circ}$.

Tampoco referían alteraciones neurológicas (a excepción del signo de Tinel en el canal epitroclear) que indiquen síndrome del túnel cubital o síndrome de compresión del nervio interóseo anterior.

Aunque no era el objetivo de nuestro estudio observamos secuelas en el codo como la disminución de la extensión y valgo, que era casi constante, aunque si revisamos en la literatura estudios comparativos con la artroplastia de sustitución, vemos como Pribyl et al. ${ }^{19}$ estudiaron la resistencia al valgo forzado de una prótesis de silastic que sustituía a la cabeza radial y vieron que era un $22 \%$ menor que en un codo intacto. Además se han descrito complicaciones en implantes de silicona tales como fracturas, sinovitis, linfadenitis, reabsorción subcondral y degeneración cartilaginosa $^{25}$. En cuanto a las prótesis de sustitución me- 
tálicas, también encontramos estudios que alertan acerca de la osteolísis progresiva en las prótesis de tipo bipolar y sus posibles repercusiones sobretodo en los pacientes más activos ${ }^{26}$.

Por todo esto podemos concluir que la extirpación de la cabeza radial no es una mala opción quirúrgica en fracturas aisladas de cabeza radial en las que sea imposible la fijación interna, y que las secuelas a medio y largo plazo en la articulación radiocubital distal no son tan importantes, o al menos no suponen tanta discapacidad para los pacientes. En la literatura encontramos estudios similares al nuestro ${ }^{20}$ que coinciden en encontrar disminución de fuerza de prensión (aproximadamente el $75 \%$ respecto al contralateral) y del arco de movimientos de la muñeca (manteniendo una media de flexoextensión mayor de $120^{\circ}$ ) que no se traducen clínicamente en importantes incapacidades laborales ya que el $90 \%$ de los pacientes retomaron su actividad habitual. En cuanto a las alteraciones radiológicas refieren una ausencia de cambios degenerativos graves en muñeca. Nuestro mayor tiempo de seguimiento quizás explica el 15,5\% de nuestra serie a pesar de una varianza ulnar o índice radiocubital distal mayor $(1,43 \mathrm{~mm}$ frente a $1,18 \mathrm{~mm}$ de media de nuestro estudio), todo esto en un $90 \%$ de pacientes asintomáticos. Es destacable que en nuestro estudio no hemos incluido fracturas más complejas del tipo Essex-Lopresti, en las que casi con certeza no hubiéramos obtenido tan buenos resultados funcionales en la articulación radiocubital distal, y para las cuales existen el la actualidad materiales específicos que facilitan la osteosíntesis y una gama considerable de prótesis rígidas. Es en este último punto donde consideramos que son necesarios amplios estudios comparativos y a largo plazo de las nuevas prótesis de sustitución que demuestre la superioridad de unas u otras.

\section{AGRADECIMIENTO}

\author{
Al Dr. Gustavo Górriz Arias \\ Jefe de sección de Cirugía plástica \\ del Hospital Donostia
}

1. Van Glabbeek F, Van Riet R, Verstreken J. Current concepts in the treatment of radial head fractures in the adult. A clinical and biomechanical approach. Acta Orthop Belg, 2001; 67: 430-41.

2. likeda M, Yamashina $Y$, Kamimoto $\mathrm{M}$, et al. Open reduction and internal fixation of comminuted fractures of the radial head using low-profile miniplates. J Bone Joint Surg Br, 2003; 85: 1040-4.

3. Parasa RB, Maffulli N. Surgical management of radial head fractures. J Royal Coll Surg Edinburgh, 2001; 46: 76-85.

4. Furry KL, Clinkscales CM. Comminute fractures of radial head.arthroplasty versus internal fixation. Clin Orthop, 1998; 353: 40-52.

5. Boulas HJ, Morrey BF. Biomechanical evaluation of the elbow following radial head fracture. Comparison of open

\section{BIBLIOGRAFÍA}

reduction aand internal fixation vs. excision, silastic replacemente and non-operative management. Chir Main, 1998; 17: 314-20.

6. King GJ, Evans DC, Kellam JF. Open reduction and internal fixation of radial head fractures. J Orthop Trauma, 1991; 5: 21-8.

7. Sanders RA, French HG. Open reduction and internal fixation of comminuted radial head fractures. Am J Sports Med, 1986; 14: 130-5.

8. Herbertsson P, Josefsson PO, Hasserius R, et al. Fractures of the radial head and neck treated with radial head excision. $\mathrm{J}$ Bone Joint Surg Am, 2004; 86: 1925-30.

9. Sánchez-Sotelo J, Romanillos O, Garay EG. Results of acute escisión of the radial head in elbow radial head fracturresdislocations. J Orthop Trauma, 2000; 14: 354-8.
10. Broberg MA, Morrey BF. Results of delayed excision of radial head after fracture. J Bone Joint Surg Am, 1986; 68: 669-74.

11. Frank SG, Grewal R, Johnson $\mathrm{J}$, et al. Determination of correct implant size in radial head arthroplasty to avoid overlengthening. J Bone Joint Surg Am, 2009; 91 : 1738-46.

12. Ring D, King G. Radial head arthroplasty with a modular metal spacer to treat acute traumatic elbow instability: Surgical technique. J Bone Joint Surg Am, 2008;. 90 Suppl 2: 63-73.

13. Dotzis A, Cochu G, Mabit C. Comminuted fractures of the radial head treated by the Judet floating radial head prosthesis. J Bone Joint Surg Br, 2006; 88: 760-4.

14. Jensen SL, Olsen BS, Sojbjerg JO. Elbow joint cinematics after excisión of the radial head. J Shoulder Elbow Surg, 1999; 8: 238-41. 
15. Morrey BF, Tanaka S, An KN. Valgus stability of the elbow. A definition of primary and secondary constraints. Clin Orthop, 1991; 265: 187-95.

16. Hall JA, McKee MD. Posterolateral instability of the elbow following radial head resection. J Bone Joint Surg Am, 2005; 87: 1571-9.

17. Johnson JA, Beingessner DM, Gordon KD, et al. Kinematics and stability of the fractured and inplant-reconstructed radial head. J Shoulder Elbow Surg, 2005; 14 (1 Suppl S): 195S-201S.

18. Hammacher ER, van der Wercken C. Radial head fractures: operative or conservative treatment?. The Greek temple model. Acta Orthop Belg, 62 Suppl 1:112-5.
19. Pribyl CR, Kester MA, Cook SD, et al. The effect of the radial head and prosthetic replacement on resisting valgus stress at the elbow. Orthopedics, 1986; 9: 723-26.

20. Miralles FA, Sebastian E, Cebrian $R$ et al. Resultados funcionales de las resecciones de la cabeza radial tras su fractura. Rev Ortop Traumatol, 2004; 48: 12-6.

21. Hotchkiss Mn, An KN, Sowa DT, et al. An anatomic and mechanical study of the interosseous membrane of the forearm: pathomechanics of proximal migration of the radius. J Hand Surg Am, 1989; 14 (2 Pt 1): 256-61.

22. Geel CW, Palmer AK. Radial head fractures and their effect on the distal radioulnar joint. $A$ rationale for treatment. Clin Orthop, 1992; 275: 79-84.
23. Crawford GP. Late radial tunnel syndrome after excision of the radial head. A report of two cases. J Bone Joint Surg Am, 1988; 70: 1416-18.

24. Broberg MA, Morrey BF. Results of delayed excision of the radial head after fracture. $\mathrm{J}$ Bone Joint Surg Am, 1986; 68: 669-74.

25. Vanderwilde RS, Morrey BF, Melberg MW, et al. Inflammatory arthritis after failure of silicone rubber replacement of the radial head. J Bone Joint Surg $\mathrm{Br}, 1994$; 76: 78-81.

26. Popovic N, Lemaire R, Georis $P$, et al. Midterm results with a bipolar radial head prosthesis: Radiographic evidence of loosening at the bone-cement interface. J Bone Joint Surg Am, 2007: 89: 2469-76. 